Research Paper

\title{
chr19_34670, a newly identified miRNA, represses rapid tumor growth of cystic vestibular schwannomas by targeting transforming growth factor $\alpha$ and inhibiting MAPK pathway
}

\author{
Zirong Huo ${ }^{1,2,3, *}$, Shuang Yan ${ }^{1,2,3, *}$, Tao Yang ${ }^{1,2,3}$, Zhentao Wang ${ }^{1,2,3}$, Qi Huang ${ }^{2,3}$, \\ Zhaoyan Wang ${ }^{1,2,3}$, Hongsai Chen ${ }^{1,2,3}$, Penghui Chen ${ }^{1,2,3}$, Huan Jia ${ }^{1,2,3}$, Zhihua \\ Zhang $^{1,2,3}$ and Hao Wu ${ }^{1,2,3}$ \\ ${ }^{1}$ Department of Otolaryngology-Head \& Neck Surgery, Shanghai Ninth People's Hospital, Shanghai Jiaotong University \\ School of Medicine, Shanghai, China \\ ${ }^{2}$ Ear Institute, Shanghai Jiaotong University School of Medicine, Shanghai, China \\ ${ }^{3}$ Shanghai Key Laboratory of Translational Medicine on Ear and Nose Diseases, Shanghai, China \\ *These authors have contributed equally to this work \\ Correspondence to: Zhihua Zhang, email: zhihua.zhang2015@aliyun.com \\ Hao Wu, email: wuhao622@sina.cn \\ Keywords: miRNA; cystic vestibular schwannoma; proliferation; tumor growth; pathogenesis \\ Received: March 16, $2017 \quad$ Accepted: July 29, $2017 \quad$ Published: January 02, 2018 \\ Copyright: Huo et al. This is an open-access article distributed under the terms of the Creative Commons Attribution License 3.0 \\ (CC BY 3.0), which permits unrestricted use, distribution, and reproduction in any medium, provided the original author and source \\ are credited.
}

\section{ABSTRACT}

Purpose: Cystic vestibular schwannoma (CVS) is an aggressive form of vestibular schwannoma (VS) with rapid growth and poor outcomes. The molecular basis of CVS remains unclear. In this study, we aimed to investigate whether microRNAs (miRNAs) contribute to the development of CVS.

Methods: We identified miRNAs that were differentially expressed between CVS and solid VS (SVS) tissues using high-throughput sequencing and quantitative real-time PCR analysis. Gene targets of the identified miRNA were predicted by bioinformatics tools and validated by luciferase reporter analysis. The effects of the miRNA and its target genes on the proliferation of primary VS cells were examined using CCK-8, flow cytometry, colony formation assay, and western-blot.

Results: We identified a novel miRNA, chr19_34670, downregulated in CVS compared to SVS. chr19_34670 directly targeted the $3^{\prime}$ untranslated region of transforming growth factor $\alpha$ (TGF $\alpha)$ mRNA and suppressed its expression. TGF $\alpha$ overexpression motivated VS cell proliferation, while this effect could be attenuated by chr19_34670. We also found that chr19_34670 inhibited the function of key proteins in the MAPK pathway by suppressing phosphorylation of ERK and MEK.

Conclusions: chr19_34670 suppresses the aggressive proliferation observed in CVS by inhibiting TGF $\alpha$ expression and phosphorylated proteins in the MAPK pathway.

\section{INTRODUCTION}

Vestibular schwannoma (VS) is one of the most common intracranial tumors in the lateral skull base that arises from Schwann cells of vestibular nerve [1]. Although histologically benign, VS can lead to hearing loss, tinnitus, vestibular and trigeminal nerve dysfunction, which significantly reduces patients' quality of life. If left untreated, VS can eventually become life threatening.

VS can be classified into two subtypes depending on their neuroradiological appearance: solid vestibular schwannoma (SVS) and cystic vestibular schwannoma 
(CVS) [2]. CVS is much more formidable than SVS due to its aggressive clinical features, such as rapid tumor growth, intensive adherence to facial nerve, more severe symptoms and unpredictable biological behaviors $[3,4]$. At present, the most common treatment approaches for VS include observation (serial imaging), microsurgical resection, and stereotactic radiosurgery $[5,6]$. However, in case of CVS, treatment becomes more difficult: conservative observation may delay the optimal treatment time; radiotherapy increases the risk of tumor expansion and recurrence; and microsurgical removal could hardly achieve satisfactory postoperative functional outcomes [7, 8].

VS typically results from the genetic disorder of neurofibromatosis type 2 (NF2) gene, which encodes the tumor suppressor protein "merlin" [9, 10]. Loss/ downregulation of $N F 2$ has also been implicated in malignant tumors, including gliomas [11], peripheral nerve sheath tumors [12] and mesotheliomas [13]. However, despite extensive studies of NF2 mutations in VS, our previous work revealed that $N F 2$ dysregulation is not directly involved in cystic formation of VS [14]. Indeed, we found no significant differences in NF2 mutations between CVS and SVS, and the proportion of mutation types (i.e., truncating and non-truncating mutations) was also similar between the two subgroups [14]. Therefore, although NF2 mutation is critical in VS pathogenesis [15], and is causative in $\sim 66 \%$ of cases [16], it may not directly participate in the development of CVS. In consequence, there is a pressing need to understand the molecular mechanisms of CVS.

Recent studies suggested that microRNAs (miRNAs) play an important role in the development and progression of VS. miRNAs comprise a class of small (approximately 20-22 nucleotides) endogenous non-coding RNAs important for many physiological and pathological processes, such as cell cycle control, cell proliferation, metastasis, apoptosis and drug resistance [17-19]. miRNAs inhibit gene expression via binding to the 3' untranslated regions (3'UTR) of target mRNAs, resulting in their translational repression or degradation [20]. When aberrantly expressed, miRNAs act as tumor suppressors or oncogenes in various benign and malignant tumors [21]. To date, several miRNAs have been implicated by microarray to be dysregulated in VS compared to control nerve sheaths. For instance, overexpression of miR-21 promotes VS growth by downregulating PTEN [22]; miR-7 functions as a tumor suppressor by targeting the EGFR, Pak1 and Ack2 oncogenes; and let-7d (a let7 family member) is upregulated in both schwannomas and meningiomas [23, 24]. Furthermore, recently, evidence showed global upregulation of the $14 \mathrm{q} 32$ chromosomal site, which contains miRNAs associated with VS formation and/or maintenance [25]. However, no evidence of a functional role for miRNAs in CVS has been documented to date.
In the present study, we investigated the differences in miRNA profiles between CVS and SVS by deep sequencing, and identified a novel miRNA, chr19_34670, which was decreased in patients with CVS.

\section{RESULTS}

\section{chr19_34670 is downregulated in CVS compared with SVS}

Twelve representative tumor specimens (six CVSs and six SVSs) were subjected to differential miRNA expression analysis using high-throughput sequencing. A hierarchical cluster of significantly upregulated or downregulated miRNAs in CVS is presented in Figure 1A. Among the nine differentially expressed miRNAs based on a fold change $\geq 2$ and $p \leq 0.05$, two were downregulated and seven were upregulated (Figure 1B).

We validated the two miRNAs downregulated in CVS using qRT-PCR in 36 tumor tissue samples of CVS compared to 32 SVSs. While chr19_34670 levels were significantly decreased in CVS $(\mathrm{p}<0.001)$, chr3_8281 levels had no statistical differences between the two groups (Figure 1C). The expression level had a statistical significant correlation $\left(\mathrm{R}^{2}=0.498 ; \mathrm{p}=0.01\right)$ between those measured by miRNA high-throughput sequencing and qRT-PCR (Figure 1D).

\section{chr19_3467 is correlated with more severe clinical characteristics}

The relationship between chr19_34670 expression level and clinical characteristics of patients diagnosed with VS (36 CVSs and $32 \mathrm{SVSs}$ ) is shown in Table 1. Then multiple linear regressions were conducted. More patients were diagnosed with CVS in the group with low chr19_34670 expression levels compared with high expression levels $(\beta=0.451, \mathrm{p}<0.001)$. Larger tumor size indicated a lower chr19_34670 expression level $(\beta=$ $-0.302, p=0.004$ ). Disease duration (from onset of disease to final diagnosis) positively correlated with chr19_34670 level $(\beta=0.264, p=0.012)$. Gender, age, post-operative facial nerve $(\mathrm{FN})$ function, and hearing level had no significant relationship with chr19_34670. Collectively, these results indicate that patients with lower chr19_34670 are more likely to be diagnosed with CVS, and thus, accompanied by more severe clinical features.

\section{chr19_34670 inhibits proliferation of primary VS cells}

We established primary VS cell models transiently transfected with a chr19_34670 mimic or inhibitor (Figure 2A) to investigate their functions in VS progression. Then we evaluated viability of primary VS cells by CCK-8 assay. As shown in Figure 2B, chr19_34670 
decreased the growth of primary VS cells compared with control group (NC mimic). In the colony formation assay, chr19_34670 overexpressing cells exhibited fewer colonies (Figure 2D). Flow cytometry analysis indicated that chr19_34670 overexpression resulted in an elevation of cells in $\mathrm{G} 1$ phase and a reduction of those in G2/M phase (Figure 2F). Conversely, decreased chr19_34670 promoted cell growth (Figure 2C) and colony formation (Figure 2E). Downregulation of chr19_34670 also increased the proportion of cells in G2/M phase (Figure $2 \mathrm{G})$. Untreated primary VS cells are used as blank controls (Supplementary Figure 1). These results suggest that chr19_34670 inhibits proliferation of primary VS cells.

\section{TGF $\alpha$ is a direct target of chr19_34670}

To explore the mechanism of chr19_34670 in primary VS cells, we investigated its potential gene targets using target prediction programs including TargetScan, miRanda, and PicTar. Among all potential target genes, TGF $\alpha$, VEGF, and NOTCH3 were previously found to be involved in cell proliferation. However, among those, only TGF $\alpha$ was upregulated in CVS compared with SVS at both mRNA and protein level (Figure 3E and 3F), and was negatively correlated with chr19_34670 levels (Figure $3 \mathrm{G})$. We further investigated the level of EGFR, the

A

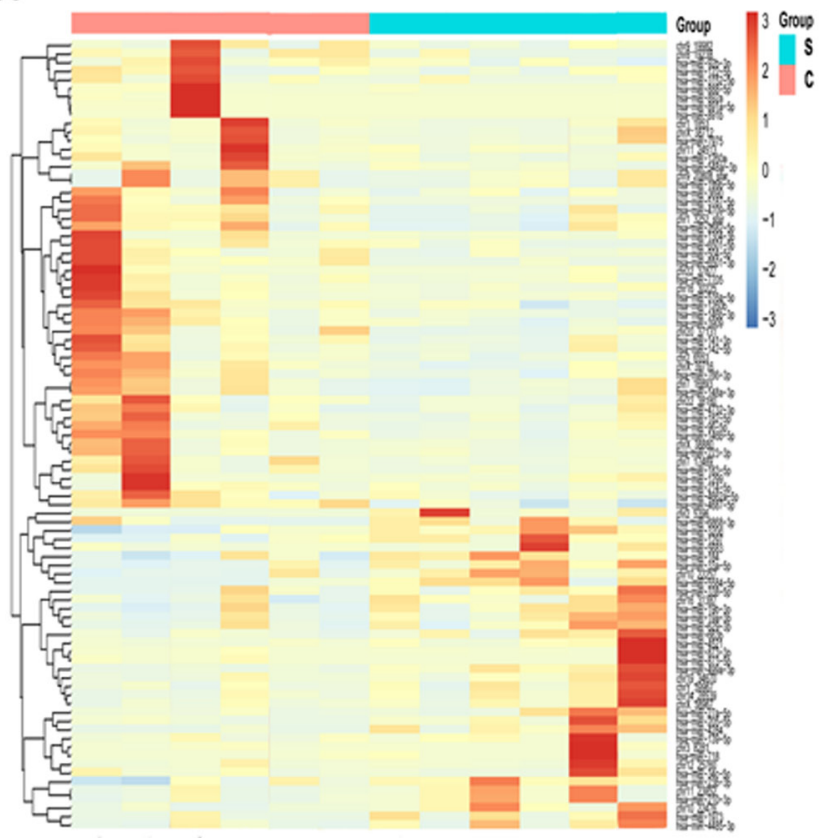

epicellular receptor of TGF $\alpha$. Interestingly, mRNA level of EGFR and protein level of p-EGFR were also increased in tumor samples of CVS (Figure 3E and 3F).

To verify whether TGF $\alpha$ is a direct target of chr19_34670, human TGFa 3'UTR fragments containing wild type (wt) or mutant (mut) chr19 34670-binding site were inserted downstream of the luciferase open reading frame (Figure 3A). These reporter constructs were cotransfected with chr19_34670 or control miRNA (NC mimic). As shown in Figure 3B, the relative luciferase activity of reporter containing wt TGF $\alpha$ 3'UTR was markedly decreased upon chr19_34670 co-transfection, while the luciferase activity of reporter containing mut chr19_34670-binding site was unaffected. Additionally, we detected mRNA and protein expression level of TGF $\alpha$ and EGFR in primary VS cells with the presence of chr19_34670 mimic or inhibitor. TGF $\alpha$ and EGFR mRNA levels were markedly reduced when chr19_34670 mimic was co-transfected, while increased with chr19_34670 inhibitor (Figure 3C). As shown in Figure $3 \mathrm{D}$, protein levels of TGF $\alpha$, EGFR and p-EGFR were also significantly decreased with the presence of chr19_34670 mimic, and increased with chr19 34670 inhibitor. These results suggest that TGF $\alpha$ is a direct target of chr19_34670 in primary VS cells.

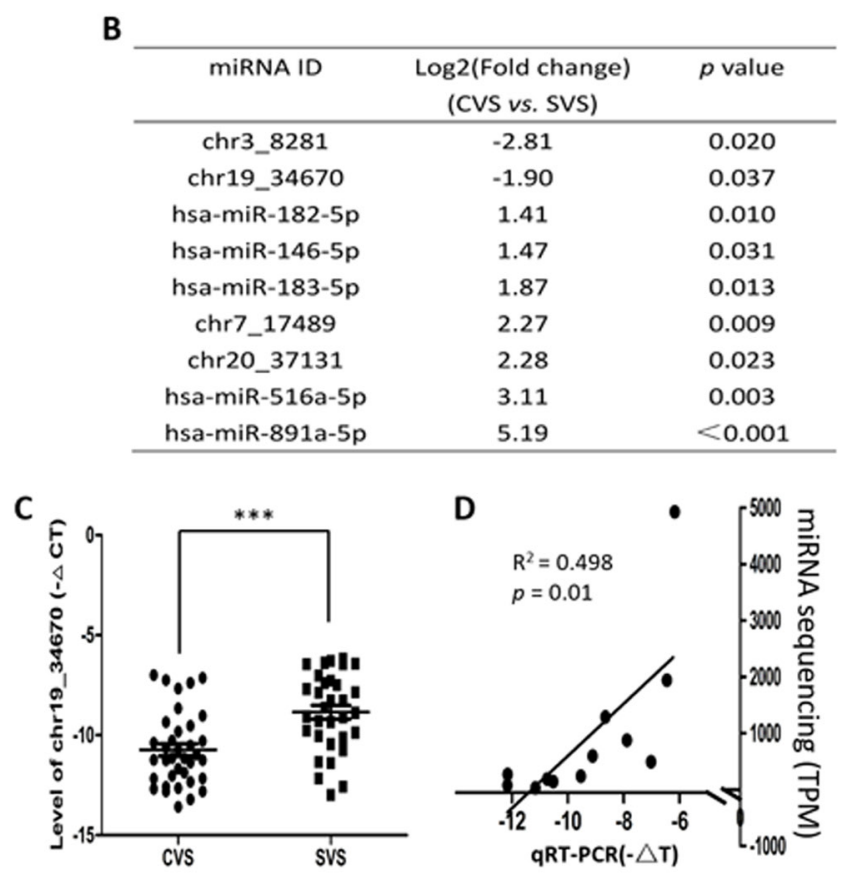

Figure 1: The expression of chr19_34670 is decreased in the tissue specimens of cystic vestibular schwannoma (CVS) compared with solid vestibular schwannoma (SVS). (A) Heat map of the differentially expressed miRNAs in CVS and SVS. (B) Differentially expressed miRNAs in CVS and SVS (adjusted $p$ values are all $<0.05$ ). (C) chr19_34670 in CVS tissues is decreased compared with SVS using qRT-PCR. (D) The consistency of miRNA sequencing and qRT-PCR is validated by linear regression. ${ }^{* * *} p<$ 0.001 . 
Table 1: Relationship between chr19_34670 expression and clinical features of VS patients

\begin{tabular}{|c|c|c|c|}
\hline \multirow{2}{*}{ Clinical features } & \multicolumn{2}{|c|}{ Relative chr19_34670 expression } & \multirow{2}{*}{$p$ value } \\
\hline & Low $(n=35)$ & High $(n=33)$ & \\
\hline \multicolumn{4}{|l|}{ Gender } \\
\hline Male & 17 & 18 & 0.622 \\
\hline Female & 18 & 15 & \\
\hline $\begin{array}{l}\text { Age, yr } \\
\text { (Range, yr) }\end{array}$ & $\begin{array}{c}46.77 \pm 13.11 \\
\quad(22-68)\end{array}$ & $\begin{array}{c}43.82 \pm 14.39 \\
\quad(11-65)\end{array}$ & 0.379 \\
\hline \multicolumn{4}{|l|}{ Tumor nature } \\
\hline CVS & 26 & 10 & $<0.001^{*}$ \\
\hline SVS & 9 & 23 & \\
\hline \multicolumn{4}{|l|}{ Tumor size (cm) } \\
\hline$\leq 3$ & 15 & 27 & $0.001^{*}$ \\
\hline$>\mathbf{3}$ & 20 & 6 & \\
\hline $\begin{array}{l}\text { Duration of symptoms, yr } \\
\text { (Range, yr) }\end{array}$ & $\begin{array}{c}3.21 \pm 3.02 \\
(0.33-14)\end{array}$ & $\begin{array}{l}4.19 \pm 3.97 \\
(0.17-20)\end{array}$ & 0.255 \\
\hline \multicolumn{4}{|l|}{ Post-op FN function } \\
\hline HB grade I-II & 26 & 29 & 0.232 \\
\hline HB grade III-IV & 7 & 4 & \\
\hline HB grade V-VI & 2 & 0 & \\
\hline \multicolumn{4}{|l|}{ Hearing classification } \\
\hline Class A & 1 & 3 & 0.744 \\
\hline Class B & 5 & 5 & \\
\hline Class C & 22 & 19 & \\
\hline Class D & 7 & 6 & \\
\hline
\end{tabular}

For chr19_34670, low and high refer to the value of $-\Delta \mathrm{T}\left(-\left(\mathrm{CT}_{\text {chr19 34670 }}-\mathrm{CT}_{\mathrm{U} 6}\right)\right)$ of qRT-PCR that are below and above the median intensity.

Values are means $\pm \mathrm{SD}$ and ranges or numbers with percentages in parentheses.

The facial nerve function was assessed according to the House-Brackmann (HB) grading system 1 year after operation, and the hearing level was assessed according to the American Academy of Otolaryngology-Head and Neck surgery (AAOHNS) classification.

CVS: cystic vestibular schwannoma; SVS: solid vestibular schwannoma; FN: facial nerve. *: $\mathrm{p}<0.05$

\section{TGFo is involved in chr19_34670-mediated cell proliferation}

To evaluate the role of TGF $\alpha$, primary VS cells were transfected with a plasmid overexpressing TGF $\alpha$ or infected with lentivirus that contained plasmid expressing TGF $\alpha$ shRNA (LV-shTGF $\alpha$ ), and their respective controls. Three days after transfection/infection, protein levels of TGF $\alpha$, EGFR, and p-EGFR were significantly increased in cells transfected with the TGF $\alpha$ overexpressing plasmid (Figure
4A), but decreased in cells infected with LV-shTGF $\alpha$ (Figure 4B). CCK-8 analysis revealed that TGF $\alpha$ overexpression significantly promoted cell growth $(\mathrm{p}<0.001)$, while the empty vector had no effect on cell growth (Figure 4C). Compared with control group, primary VS cells overexpressing TGF $\alpha$ had an increased proportion of cells in $\mathrm{G} 2 / \mathrm{M}$ phase (Figure $4 \mathrm{E}$ ), and formed more colonies ( $\mathrm{p}$ $=0.002$; Figure $4 \mathrm{G}$ ). Conversely, cells infected with LVshTGFa grew much slower in CCK- 8 assay $(\mathrm{p}<0.001$; Figure 4D), had smaller proportion of cells in $\mathrm{G} 2 / \mathrm{M}$ phase 
(Figure 4F), and formed fewer colonies than control group $(p=0.009$; Figure 4H). When chr19_34670 mimic was co-transfected into primary VS cells with the plasmid overexpressing TGF $\alpha$, these TGF $\alpha$-mediated effects were attenuated (Figure 4C $[\mathrm{p}=0.006]$, Figure 4E and 4G $[\mathrm{p}=$ 0.003]. These findings suggest that chr19 34670 inhibits VS cell proliferation by suppressing TGF $\alpha$ expression.

\section{chr19_34670 suppresses phosphorylation of ERK/MEK}

To reveal possible mechanisms of chr19_34670 and TGF $\alpha$ in proliferation of primary VS cells, we detected the expression levels of Akt, p-Akt, and the proteins associated with MAPK signaling pathway, such as ERK1/2, p-ERK1/2, MEK1/2, and p-MEK1/2. chr19_34670 mimic inhibited the expression of p-MEK1/2, ERK1/2, and p-ERK1/2 compared with NC mimic, whereas levels of these three proteins increased with the presence of chr19_34670 inhibitor (Figure
5A). Western blot analysis also showed that TGF $\alpha$ promoted cell proliferation by increasing the expression of $\mathrm{p}-\mathrm{MEK} 1 / 2$, ERK1/2, and p-ERK1/2 (Figure 5B). The protein levels of p-MEK1/2, ERK1/2, and p-ERK1/2 were largely increased in primary VS cells transfected with TGF $\alpha$ overexpressing plasmid. This effect could be attenuated upon co-transfection with chr19 34670 mimic (Figure 5B). On the contrary, p-MEK1/2, ERK1/2 and p-ERK1/2 were decreased when LV-shTGF $\alpha$ was infected into VS primary cells (Figure 5C). The expression level of other proteins, including Akt, p-Akt and MEK, showed no significant differences. These findings suggest that chr19_34670 represses proliferation by inhibiting key proteins in MAPK signaling pathway.

\section{DISCUSSION}

To our knowledge, this is the first study comprehensively investigating the differences in miRNA profiles between sporadic CVS and SVS using
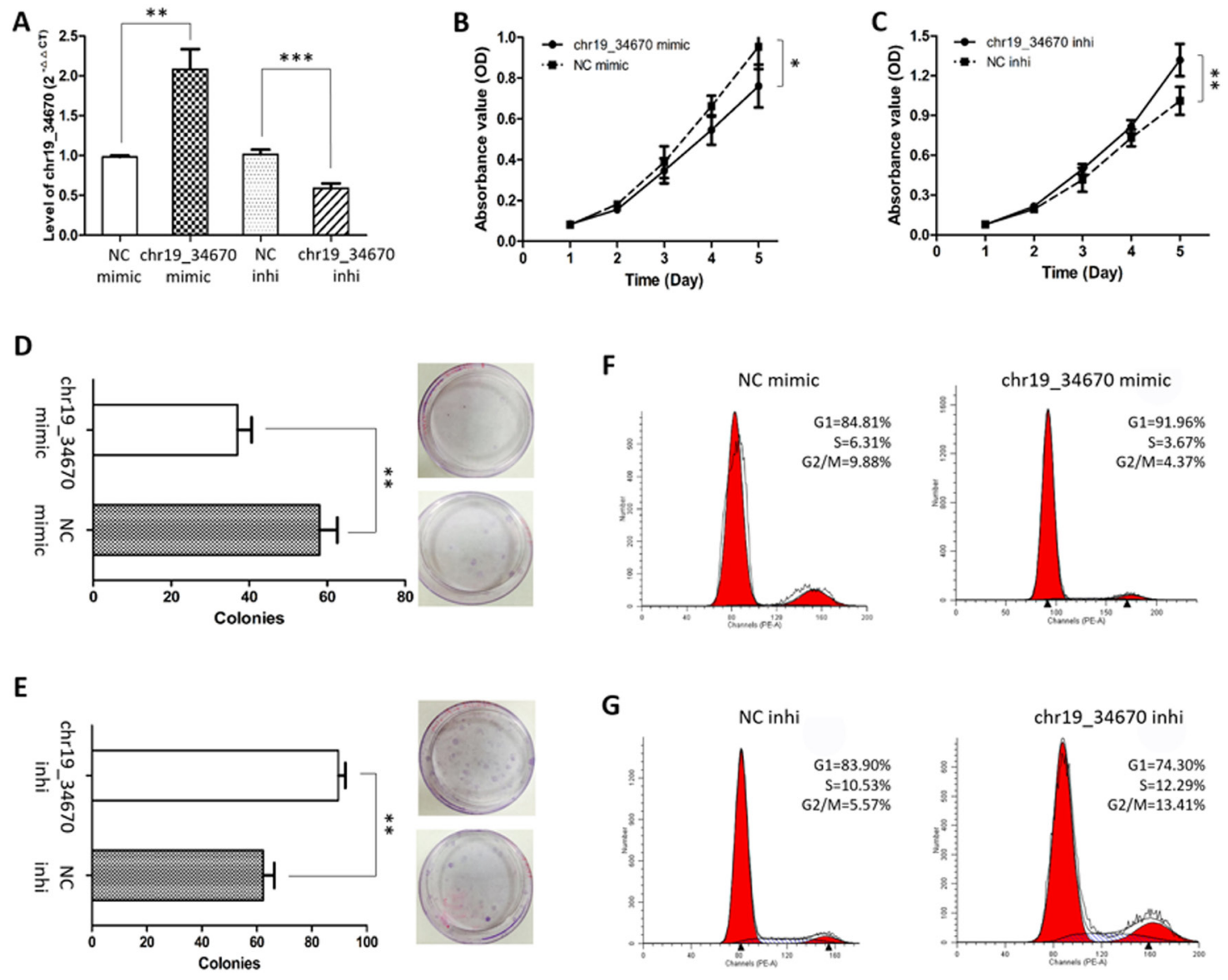

Figure 2: Phenotypes resulting from upregulation and downregulation of chr19_34670. (A) The expression level of chr19_34670 in primary vestibular schwannoma (VS) cells $48 \mathrm{~h}$ after transfection with chr19_34670 mimic and inhibitor compared with their respective controls (NC). The effects on proliferation after downregulation or overexpression of chr19_34670 in primary VS cells are validated by CCK-8 assay (B, C), colony formation assay $(\mathbf{D}, \mathbf{E})$ and flow cytometry $(\mathbf{F}, \mathbf{G})$. Proliferation of VS cells with chr19_34670 overexpression is inhibited compared to control group (B, D \& F). chr19_34670 inhibitor induces proliferation of VS cells compared to control group $(\mathrm{C}, \mathrm{E} \& \mathrm{G})$. Each bar represents the mean $\pm \mathrm{SD}$. The results are reproduced in three independent experiments. ${ }^{*} p<0.05 ;{ }^{* *} p$ $<0.01 ;{ }^{* * *} p<0.001$. 
high-throughput sequencing, which is a powerful tool for analyzing the expression of candidate miRNAs or siRNAs on a large scale [26]. As chr19_34670 was downregulated in CVS (Figure 1), we speculated that overexpression of chr19_34670 might suppress VS cell proliferation and therefore inhibit cystic change of VS. Indeed, restoration of chr19_34670 in primary
VS cells significantly suppressed cell proliferation in vitro (Figure 2). Furthermore, our clinical analysis revealed a strong association between low chr19_34670 levels and more aggressive clinical features, such as large tumor size and rapid tumor growth. These results suggest chr19_34670 as a tumor suppressor in VS. Other candidate miRNAs identified in our study have been
A

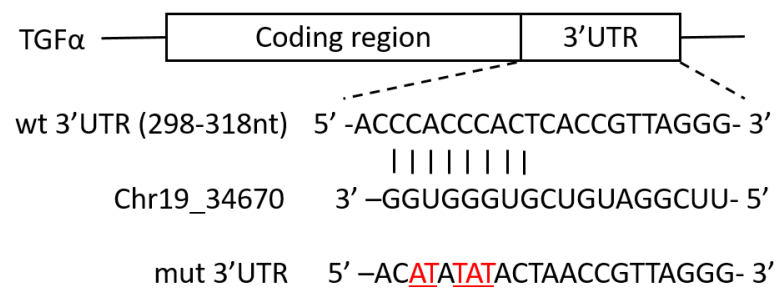

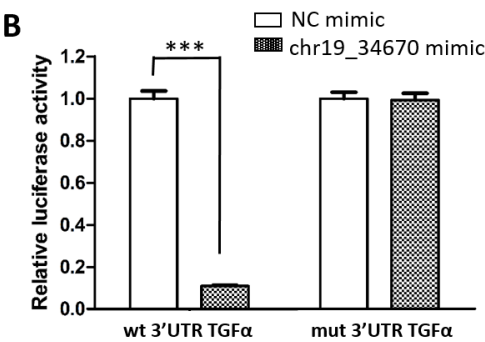

D TGF $\alpha$

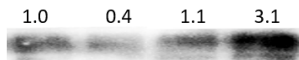

C
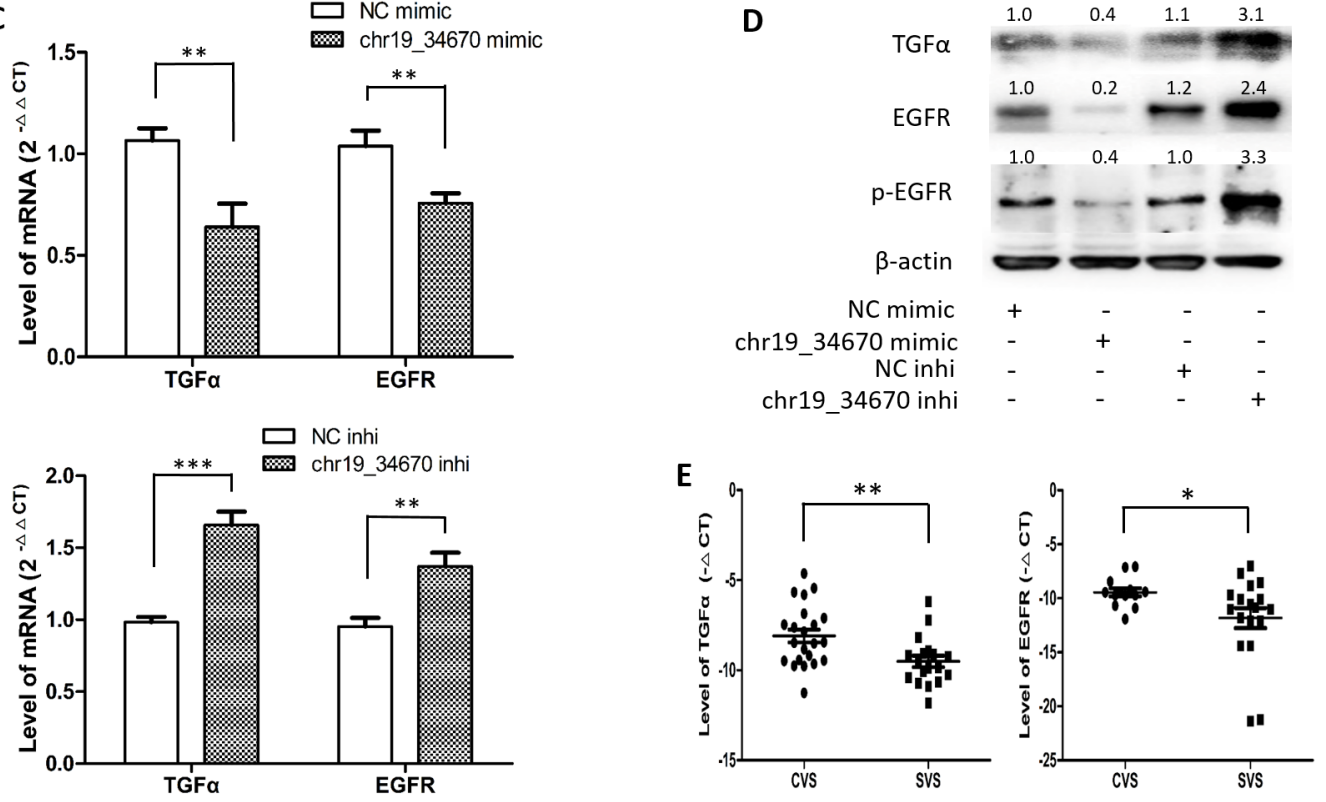

$\mathbf{F}$
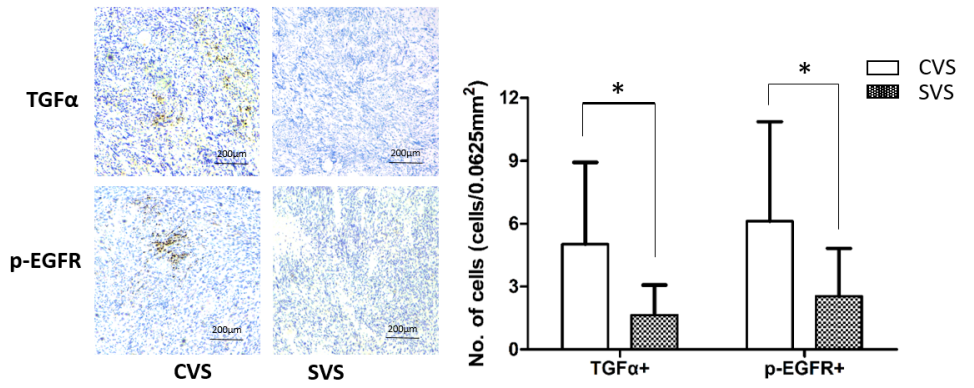

G

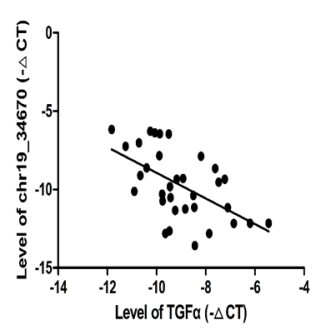

Figure 3: miRNA target gene prediction and validation. The target gene of chr19_34670 is predicted by bioinformatics tools (A) and validated by luciferase reporter analysis (B). The levels of TGF $\alpha$ and its cell surface receptor (EGFR) in primary vestibular schwannoma (VS) cells transfected with the chr19_34670 mimic/inhibitor and their respective controls (NC) are determined by qRT-PCR (C) and western blot (D). Expression levels of TGF $\alpha$ and EGFR/p-EGFR in VS tissues are determined by qRT-PCR (E) and immunohistochemistry (F). The negative correlation of chr19 34670 and TGF $\alpha$ expressed in VS tissues is verified by linear regression (G). Each bar represents the mean \pm SD. ${ }^{*} p<0.05 ;{ }^{* *} p<0.01 ;{ }^{* * *} p<0.001$. 
A

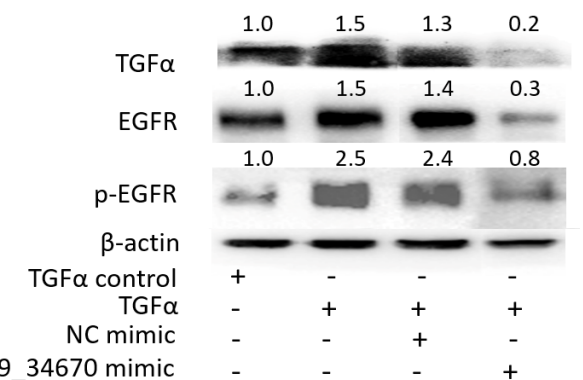

chr19_34670 mimic
B

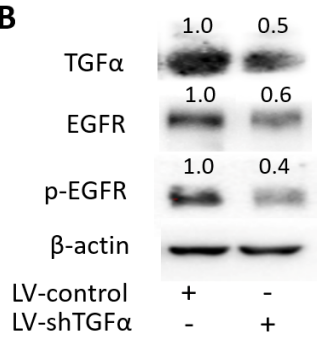

C

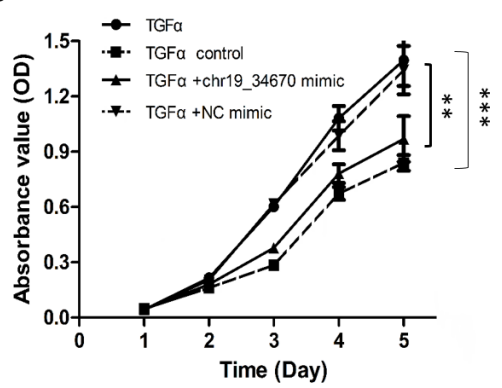

D

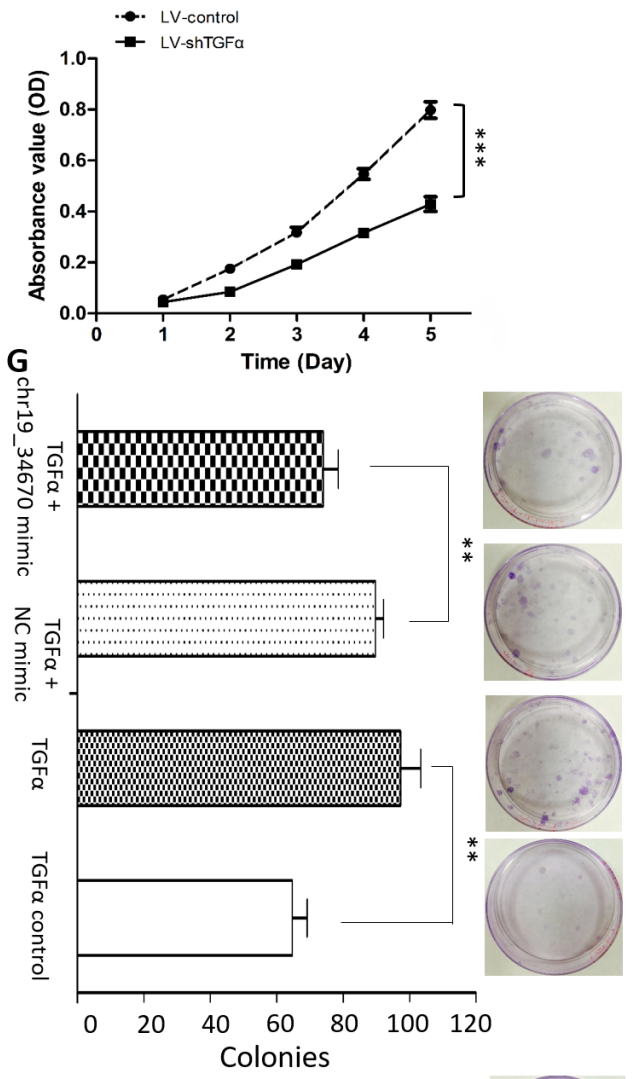

E
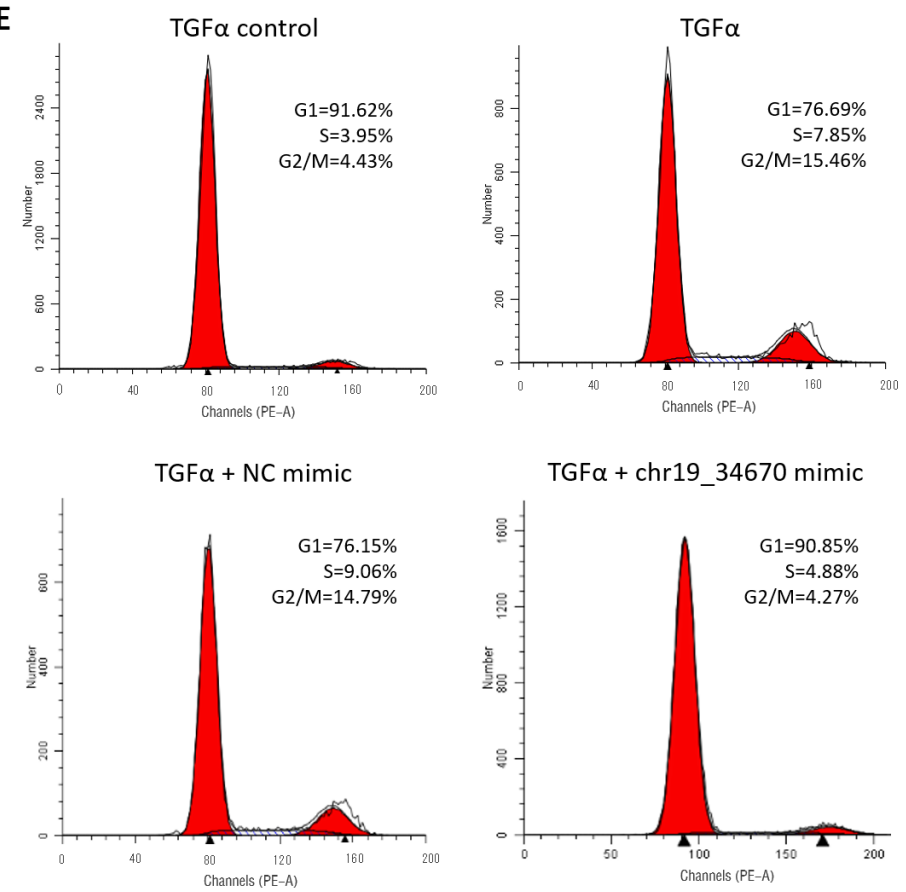

TGF $\alpha+$ chr19_34670 mimic

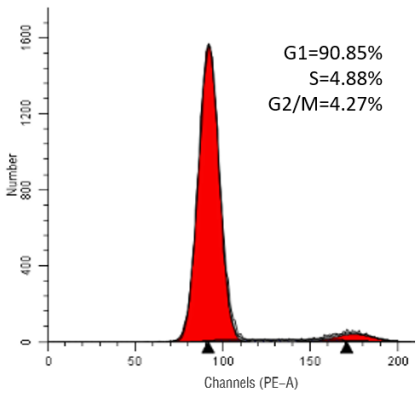

$\mathbf{F}$
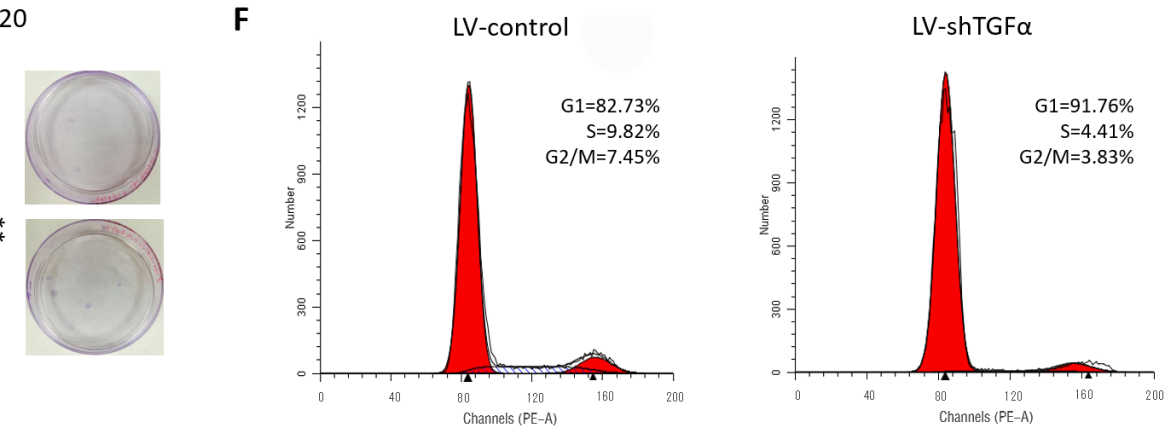

Figure 4: The effect of TGF $\alpha$ and chr19_34670 on proliferation of primary VS cells. There are four groups in figure (A, $\mathbf{C}, \mathbf{E}$ and $\mathbf{G})$ primary vestibular schwannoma ( $\overline{\mathrm{VS}})$ cells transfected with TGF $\alpha$ overexpressing plasmid, empty plasmid (TGF $\alpha$ control), co-transfected with TGF $\alpha$ overexpressing plasmid and chr19_34670 mimic or miRNA control mimic (NC mimic). In figure (B, D, F and $\mathbf{H}$ ) two groups can be seen: primary VS cells infected with lentivirus expressing shTGF $\alpha$ (LV-shTGF $\alpha$ ) or empty lentivirus (LVcontrol). Expression levels of TGF $\alpha$ and its receptor EGFR/p-EGFR are examined by western blot 3 days after incubation (A, B). In vitro proliferation of primary VS cells is measured by CCK-8 (C, D), flow cytometry (E, F), and colony formation assay (G, H). Each bar represents the mean $\pm \mathrm{SD}$. The experiments are performed at least three times. ${ }^{* *} p<0.01 ;{ }^{* * *} p<0.001$. 
previously documented with the indicated capabilities in various tumors. For instance, miR-183 was found to be decreased in melanomas and schwannomas compared with local normal tissues or normal nerves $[25,27]$. This indicates the reliability of high-throughput sequencing platform for proliferation phenotype analysis and the potential important role of miRNAs in VS cell proliferation.

We then predicted potential target genes of chr19 34670 using bioinformatics tool. To narrow down the number of target genes, we compared the candidate genes with previous result of mRNA chip for CVS and SVS. Briefly speaking, we selected the candidate genes that were up-regulated in CVS according to mRNA chip [14]. Among these genes, TGF $\alpha$, VEGF, and NOTCH3 were previously found to be involved in cell proliferation. However, only TGF $\alpha$ showed statistical difference between CVS and SVS at both mRNA and protein levels (Figure 3E and 3F).

Our present study further validated that TGF $\alpha$ was a direct target of chr19_34670 and that chr19_34670mediated inhibition of TGF $\alpha$ was dependent on a conserved motif in the 3'UTR of TGF $\alpha$ (Figure 3 ). The expression and phosphorylation levels of EGFR, the cellsurface receptor of TGF $\alpha$, were also decreased with the presence of chr19_34670 mimic. Overexpression of TGF $\alpha$ stimulated proliferation of primary VS cells, which was similar to the effects of chr19_34670 downregulation. Furthermore, chr19_34670 could rescue cell proliferation induced by $\mathrm{TGF} \alpha$ overexpression. Collectively, our data reveal that chr19_34670 exerts its tumor-suppressive function in CVS, at least in part, through downregulating TGF $\alpha$.
TGF $\alpha$ plays a pivotal part in embryonic development through increasing the expression of pluripotent markers [28]. Moreover, TGF $\alpha$ dysregulation is often associated with various cancers [29]. TGF $\alpha$ is not only an autocrine mitogen, but also a modulator of tumor microenvironment in a paracrine manner, enabling cross-talk between the tumor and its surrounding stroma and immune system [30, 31]. As TGF $\alpha$ mRNA has disproportionately large 3'UTR with multiple potential miRNA binding sites, its expression could be modulated post-transcriptionally by various miRNAs. Indeed, both miR-376c (which exhibits anticancer properties in a number of cancers including melanoma and osteosarcoma) [32] and miR152 (which functions as a tumor suppressor in prostate tissues) [33] have been shown to target TGF $\alpha$. Previous studies comparing the miRNA expression profiles of VS to control nerve tissues identified a schwannomatypical signature [24]. In addition, they found that miR-7 inhibited schwannoma cell growth by directly targeting EGFR, the cell surface receptor of TGF $\alpha$. Although this study did not compare the differences between CVS and SVS, the results together with our present study indicate that $\mathrm{TGF} \alpha / \mathrm{EGFR}$ pathway promotes VS cell proliferation.

Because TGF $\alpha$ influences multiple cell functions, it is not surprising that the proteins identified in the downstream pathway are involved in a variety of biological processes. While activation of the TGF $\alpha$ / EGFR pathway may promote invasive behavior in VS through the ERK and Akt signaling pathways [34], the physiological significance of TGF $\alpha$ in cystic variant and
A

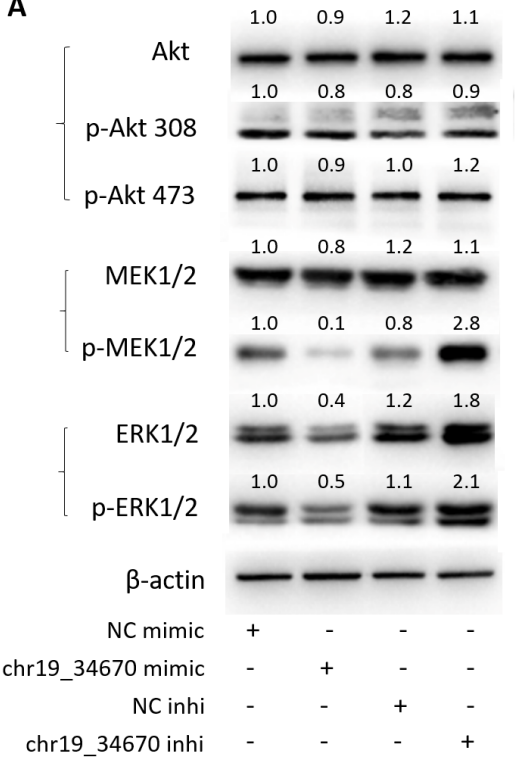

B

Akt
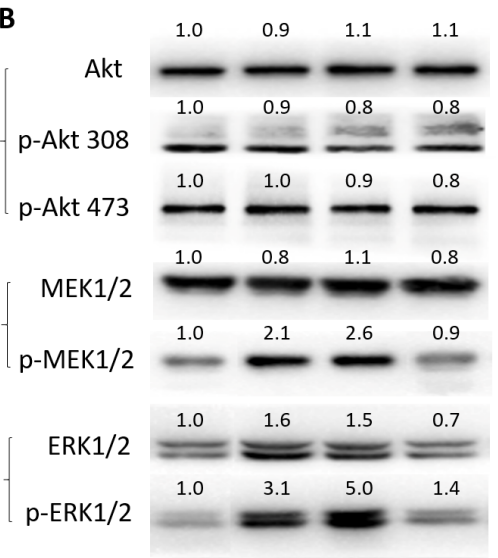

$\beta$-actin

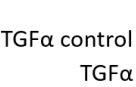

TGF $\alpha$

NC mimic

chr19_34670 mimic
C
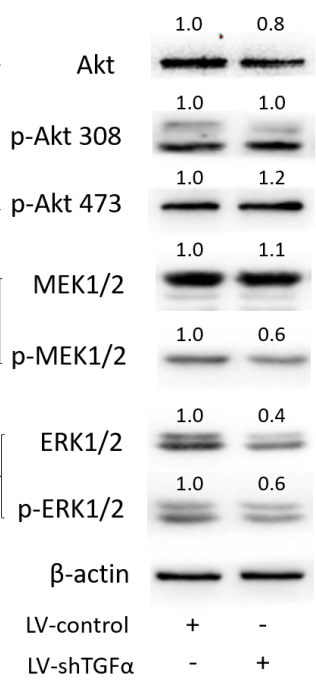

Figure 5: The underlying mechanisms of chr19_34670 and TGF $\alpha$ in the proliferation of primary vestibular schwannoma (VS) cells. Phosphorylation of Akt and MAPK signaling pathway-associated proteins (ERK1/2, MEK1/2) are detected by western blot. 
growth of VS remained elusive. Our study demonstrate that the chr19_34670/TGFa/EGFR pathway modulates the growth of VS through MAPK pathway by suppressing phosphorylation of ERK1/2 and MEK1/2, but not Akt (Figure 5). It is widely acknowledged that activation of ERK and MEK is essential in tumor development and progression [35]. Therefore, our results suggest that chr19_34670/TGF $\alpha / E G F R / M A P K$ pathway is a novel potential therapeutic target for CVS.

As cystic change is difficult to observe at the cellular level, thus we monitored the growth rate in vitro to distinguish CVS from SVS. In future, we will elucidate whether introducing chr19_34670 into VS cells can slow down tumorigenesis and inhibit cystic variant of VS in appropriate mouse models. Furthermore, this study mainly focused on miRNAs that were downregulated in CVS compared to SVS. Therefore, the role of any upregulated miRNAs in the tumorigenesis and progression of VS should also be investigated. Changchun et al [36] pointed out that transient transfection of chemically synthesized miRNA mimics may not be similar to the endogenous miRNA due to accumulation of a few hundred fold increase in mature miRNA levels. To avoid potential artifacts, we measured the level of miRNA after transfection of miRNA mimics using qRT-PCR, and control this level within 10 fold compared with controls (unrelated miRNA mimics). In addition, animal experiments performed with lentivirusinfected cells are necessary in future.

In summary, we identified a novel miRNA, chr19_34670, which inhibited the proliferation of primary VS cells by directly targeting on TGF $\alpha$ and inhibiting the MAPK signaling pathway. Our findings shed light upon the mechanisms behind rapid growth of CVS, and may provide new potential therapeutic targets for its prevention and treatment.

\section{MATERIALS AND METHODS}

\section{Patients and tumor tissue samples}

Fresh VS specimens were obtained from patients diagnosed with sporadic VS who underwent microsurgical resection at a general hospital between 2012 and 2016. The patients were classified as CVS or SVS according to preoperative neuroradiological appearance.

\section{miRNA sequencing}

Six CVS and six SVS tissue samples were cut into pieces and dipped in RNAstore Reagent (TIANGEN, Beijing, China) immediately after resection and shipped on dry ice to Jing Neng Bio-Technology corporation (Shanghai, China) for high-throughput sequencing as described previously [37]. A miRNA library of VS tissue was created by combining the sequencing data from all twelve samples. The numbers of transcripts per million
(TPM) were assessed to compare the sequences in each sample with the miRNA library established in this study. Differentially expressed miRNAs between CVS and SVS were identified using DESeq software [38] on the basis of a fold change $(\mathrm{FC}) \geq 2$ and $\mathrm{p}$ value $\leq 0.05$.

In addition, putative miRNA targets were predicted and analyzed using three publicly available algorithms: TargetScan, miRanda, and PicTar [39]. False-positives were reduced by obtaining a similar prediction with at least two programs.

\section{Primary cell culture}

Primary VS cell cultures were prepared as previously described [40]. Briefly, acutely resected tumors were cut into $1 \mathrm{~mm}^{3}$ fragments, and digested with $0.25 \%$ trypsin (Gibco, Waltham, USA) for 100-120 minutes at $37{ }^{\circ} \mathrm{C}$. Following centrifugation at $800 \mathrm{rpm}$ for 5 minutes, the cells were resuspended and dissociated by trituration through narrow pipettes. Cell suspensions were filtered through a mesh of $75 \mu \mathrm{m}$ and plated in petri dishes. Cultures were maintained in Schwann Cell Medium (SCM; ScienCell, CA, USA), and the medium was exchanged two days later. More than $90 \%$ of the cells in the cultures were S100-positive (Supplementary Figure 2 ). Cultures were maintained in a humidified incubator with $5 \% \mathrm{CO}_{2}$ at $37^{\circ} \mathrm{C}$.

\section{RNA isolation and quantitative real-time PCR (qRT-PCR)}

Forty-eight hours after transfection or after tumor tissue collection, total RNA was extracted from cells or tissues with TRIzol reagent (TaKaRa, Dalian, China). To quantify chr19_34670 expression, total RNA was polyadenylated and underwent reverse transcription (RT) with miRNA-specific RT primers (TaKaRa, Dalian, China). mRNA levels of TGF $\alpha$ and epidermal growth factor receptor (EGFR) were measured as previously described [41]. qRT-PCR was carried out using an SYBR Green PCR kit (TaKaRa, Dalian, China) on an Applied Biosystems 7500 Fast Sequence Detection System. ACTB or U6 was used as an endogenous control. The results were analyzed and shown as relative miRNA or mRNA levels of the $C_{T}$ (cycle threshold) value. Primers were purchased from Sangon Biotech, Shanghai; the primer sequences are provided in Supplementary Table 1.

\section{Construction of the reporter gene system and luciferase activity assay}

A 543-bp fragment of the TGF $\alpha$ 3'UTR was amplified by PCR and cloned downstream of the firefly luciferase gene in the pMIR vector (Invitrogen, Waltham, USA). This vector was named the wild-type (wt) 3'UTR. Site-directed mutagenesis of the chr19_34670 binding site 
in the 3'UTR of TGF $\alpha$ was carried out to create the mutant (mut) 3'UTR. Sequencing was performed by Sangon Biotech (Shanghai, China) to verify the constructs. For the reporter assays, the wt or mut 3'UTR vector and the chr19_34670 or NC mimic were co-transfected. Luciferase activity was measured 48 hours after transfection using Dual-Glo Luciferase Assay System (Promega, Madison, USA) and normalized to respective control.

\section{Immunohistochemistry}

Immunohistochemistry of tumor tissue sections was performed as described previously. Briefly, the 5- $\mu \mathrm{m}$ sections were de-waxed, rehydrated, and rinsed with phosphate-buffered saline (PBS). An antigen-demarking procedure was carried out following high temperature heating, immersion in citrate buffer $(10 \mathrm{mmol} / \mathrm{L}, \mathrm{pH} 6.0)$, and rinsing with $\mathrm{PBS}$. Endogenous peroxidases were blocked with $3 \% \mathrm{H}_{2} \mathrm{O}_{2}$ for 8 minutes, and nonspecific binding was blocked with 5\% normal goat serum for 30 minutes. Sections were then incubated for 2 hours at room temperature with rabbit monoclonal anti-EGFR or p-EGFR antibody (1:50; CST, Danvers, USA), and mouse monoclonal anti-TGF $\alpha$ antibody (1:50; LifeSpan BioSciences, Seattle, USA). After washing with PBS, sections were incubated with biotinylated secondary antibody, followed by a further incubation with the streptavidin-horseradish peroxidase complex. The sections were then immersed in 3, 3-diaminobenzidine for 10 minutes, counterstained with $10 \%$ hematoxylin, dehydrated, and photographed under a light microscope (×200 magnification).

\section{Western blot}

Seventy-two hours after transfection, cells were harvested and subjected to RIPA buffer (Beyotime, Nantong, China) with the presence of a protease inhibitor cocktail (Sigma, St. Louis, USA) and phosphatase inhibitor (PhosSTOP, Roche, Basel, Switzerland). After centrifugation at $12,000 \mathrm{~g}$ for 15 minutes at $4{ }^{\circ} \mathrm{C}$, the supernatant fraction was collected. Protein expression was quantified by standard western blot procedures with the following antibodies: TGF $\alpha$ (2.5:1000; LifeSpan BioSciences, Seattle, USA); Akt, p-Akt (Ser308), p-Akt (Ser473), $\beta$-actin (1:1000; Beyotime, Nantong, China); MAPK (ERK1/2), p-p44/42 MAPK (ERK1/2), MEK1/2, p-MEK1/2 (1:1000; CST, Danvers, USA). Protein levels were normalized to total $\beta$-actin levels. Quantitative data were obtained using a computing densitometer and GelPro Analyser 4.0 (Media Cybernetics, Maryland, USA).

\section{miRNA transfection}

The cells were cultured in SCM with $5 \% \mathrm{CO}_{2}$ at $37^{\circ} \mathrm{C}$. miRNAs were transfected at a working concentration of $100 \mathrm{nmol} / \mathrm{L}$ using Lipofectamine 3000 reagent (Lip 3000, Invitrogen, Waltham, USA). The chr19_34670 mimic, a non-specific miRNA control (NC mimic), chr19_34670 inhibitor (chr19_34670 inhi), and a non-specific miRNA inhibitor control (NC inhi) were purchased from GenePharma (Shanghai, China). The sequences are listed in Supplementary Table 2. Transfection was performed using Lip 3000 according to the manufacturer's instructions.

\section{Plasmid construction and stable transfection}

The coding sequences of TGF $\alpha$ were cloned into pCMV--Puro to generate a TGF $\alpha$ expression vector (purchased from Lqbiotech Corporation, Shanghai, China). The primers for TGF $\alpha$ were: 5'-CTAGTCTAGAGCCAC CATGGTCCCCTCGGCTGGAC-3' (forward) and 5'-TC CTTCGAAGACCACTGTTTCTGAGTGGC-3' (reverse). For stable knockdown of $\mathrm{TGF} \alpha$, short hairpin RNA (shRNA) oligos (5'-GCAGTGGTGTCTCACTTCA-3'; Asia-Vector Biotechnology, Shanghai, China) were annealed and cloned into the lentiviral expression vector pGIPZ-Puro, and the construct was named LV-shTGF $\alpha$. The empty lentiviral expression vector was named LVcontrol. The efficacy of transfection was tested by qRTPCR and western blot.

Primary VS cells were plated at a density of $1 \times 10^{5}$ cells per well in a 6-well plate the day before viral infection. Transfection of plasmid overexpressing TGF $\alpha$ and the control plasmid (empty vector) was performed using Lip 3000 according to the manufacturer's protocol. The virus diluted for infection at an MOI of 20 into 0.5 $\mathrm{ml}$ of SCM medium, and polybrene was added to a final concentration of $5 \mathrm{mg} / \mathrm{ml}$. Primary VS cells were infected by adding the viral stock dilutions to the wells.

\section{Cell proliferation assay}

Primary VS cells transfected with chr19_34670 mimic, chr19_34670 inhibitor, TGF $\alpha$ overexpression plasmid, and the respective controls, or infected with LVshTGF $\alpha$, were seeded at a density of $2 \times 10^{3}$ cells in 96-well plates and incubated for various periods of time (1 to 5 days). Following incubation, Cell Counter Kit-8 (CCK8; $10 \mu \mathrm{L}$; Yeasen, Shanghai, China) reagent was added to each well and cells were incubated at $37^{\circ} \mathrm{C}$ for 3 hours. Absorbance was measured with an electroluminescence immunosorbent assay reader (Biotek, Vermont, USA) at a wavelength of $450 \mathrm{~nm}$.

\section{Flow cytometry}

Three days after transfection or infection, floating and adherent cells were collected after centrifugation at $1000 \mathrm{rpm}$ for 10 minutes, washed with PBS, and fixed in $75 \%$ ethanol overnight at $4{ }^{\circ} \mathrm{C}$. Fixed cells were stained with propidium iodide in a PBS solution containing RNase A, and analyzed using a Calibur fluorescence-activated 
cell sorter (FACS, Becton Dickinson, Franklin Lake, USA) [42].

\section{Colony formation assay}

Briefly, cells $\left(0.5 \times 10^{3}\right)$ were plated into six-well plates and incubated at $37{ }^{\circ} \mathrm{C}$ in a humidified incubator with $5 \% \mathrm{CO}_{2}$ for 10 days. Colonies were then fixed for 20 minutes with $4 \%$ formaldehyde and stained with $1 \%$ crystal violet for 30 minutes. The number of colonies containing more than 50 cells was counted using a microscope.

\section{Statistical analysis}

Data are presented as means \pm standard deviation (SD) and analyzed with SPSS17.0 software (SPSS Inc., Chicago, IL). Analysis of variance (ANOVA) or tow-tailed Student $t$-test was used to compare the differences between two groups. Multiple linear regression was performed to find out the relationship between chr19_34670 expression level and various clinical features of patients diagnosed with VS. A $p$-value $<0.05$ was considered statistically significant. All experiments were performed at least three times.

\section{Author contributions}

Zirong Huo produced metadata, maintained research data for initial and later use, apply statistical and mathematical techniques to analyze study data, and created the initial draft. Shuang Yan collected the initial clinical data and samples, did the computer work about data analysis and figures making. Tao Yang and Zhentao Wang gave directions for development of methodology, and took the responsibility for the research activity planning and execution. Qi Huang and Zhaoyan Wang provided clinical materials and patients, validated the overall reproducibility of the experiments and all of the outputs, Zhaoyan Wang also provided foundation support. Hongsai Chen and Penghui Chen offered help with programming and algorithms necessary for data analyzing. Zhihua Zhang and Hao Wu came up with the ideas of this research, made up the research goals and aims, provided financial support, conducted a research and investigation process, took the leadership responsibility for research activity planning and execution, reviewed the paper work and carried out the final version.

\section{ACKNOWLEDGMENTS}

The authors thank the patients who participated in this study. The authors also appreciate Dr. Kun Chen and Bin Ye, who provided kind technical help and writing assistance.

\section{CONFLICTS OF INTEREST}

The authors declare no conflicts of interest.

\section{FUNDING}

This work was supported by grants from the National Natural Science Foundation of China [grant number 81470681, 81570906 and 81670919], the Shanghai Natural Science Foundation [grant number 14ZR1426600], and Shanghai Key Laboratory of Translational Medicine on Ear and Nose diseases Foundation [grant number 14DZ2260300].

\section{REFERENCES}

1. Mahaley MS Jr, Mettlin C, Natarajan N, Laws ER Jr, Peace BB. Analysis of patterns of care of brain tumor patients in the United States: a study of the Brain Tumor Section of the AANS and the CNS and the Commission on Cancer of the ACS. Clin Neurosurg. 1990; 36:347-352.

2. Piccirillo E, Wiet MR, Flanagan S, Dispenza F, Giannuzzi A, Mancini F, Sanna M. Cystic vestibular schwannoma: classification, management, and facial nerve outcomes. Otol Neurotol. 2009; 30:826-834.

3. Moon KS, Jung S, Seo SK, Jung TY, Kim IY, Ryu HH, Jin YH, Jin SG, Jeong YI, Kim KK, Kang SS. Cystic vestibular schwannomas: a possible role of matrix metalloproteinase-2 in cyst development and unfavorable surgical outcome. J Neurosurg. 2007; 106:866-871.

4. Mehrotra N, Behari S, Pal L, Banerji D, Sahu RN, Jain VK. Giant vestibular schwannomas: focusing on the differences between the solid and the cystic variants. Br J Neurosurg. 2008; 22:550-556.

5. van de Langenberg $\mathrm{R}$, de Bondt BJ, Nelemans PJ, Dohmen AJ, Baumert BG, Stokroos RJ. Predictors of volumetric growth and auditory deterioration in vestibular schwannomas followed in a wait and scan policy. Otol Neurotol. 2011; 32:338-344.

6. Muller S, Arnolds J, van Oosterhout A. Decision-making of vestibular schwannoma patients. Acta Neurochir (Wien). 2010; 152:973-984.

7. Sinha S, Sharma BS. Cystic acoustic neuromas: surgical outcome in a series of 58 patients. J Clin Neurosci. 2008; 15:511-515.

8. Zhang Z, Wang Z, Huang Q, Yang J, Wu H. Removal of large or giant sporadic vestibular schwannomas via translabyrinthine approach: a report of 115 cases. ORL J Otorhinolaryngol Relat Spec. 2012; 74:271-277.

9. Evans DG, Sainio M, Baser ME. Neurofibromatosis type 2. J Med Genet. 2000; 37:897-904.

10. Hadfield KD, Smith MJ, Urquhart JE, Wallace AJ, Bowers NL, King AT, Rutherford SA, Trump D, Newman WG, Evans DG. Rates of loss of heterozygosity and mitotic 
recombination in NF2 schwannomas, sporadic vestibular schwannomas and schwannomatosis schwannomas. Oncogene. 2010; 29:6216-6221.

11. Lau YK, Murray LB, Houshmandi SS, Xu Y, Gutmann DH, Yu Q. Merlin is a potent inhibitor of glioma growth. Cancer Res. 2008; 68:5733-5742.

12. Scheithauer BW, Erdogan S, Rodriguez FJ, Burger PC, Woodruff JM, Kros JM, Gokden M, Spinner RJ. Malignant peripheral nerve sheath tumors of cranial nerves and intracranial contents: a clinicopathologic study of 17 cases. Am J Surg Pathol. 2009; 33:325-338.

13. Sekido Y. Genomic abnormalities and signal transduction dysregulation in malignant mesothelioma cells. Cancer Sci. 2010; 101:1-6.

14. Zhang Z, Wang Z, Sun L, Li X, Huang Q, Yang T, Wu $\mathrm{H}$. Mutation spectrum and differential gene expression in cystic and solid vestibular schwannoma. Genet Med. 2014; 16:264-270.

15. Caye-Thomasen P, Borup R, Stangerup SE, Thomsen $\mathrm{J}$, Nielsen FC. Deregulated genes in sporadic vestibular schwannomas. Otol Neurotol. 2010; 31:256-266.

16. Gutmann DH, Giordano MJ, Fishback AS, Guha A. Loss of merlin expression in sporadic meningiomas, ependymomas and schwannomas. Neurology. 1997; 49:267-270.

17. Lund E, Guttinger S, Calado A, Dahlberg JE, Kutay U. Nuclear export of microRNA precursors. Science. 2004; 303:95-98.

18. Bartel DP. MicroRNAs: genomics, biogenesis, mechanism, and function. Cell. 2004; 116:281-297.

19. He L, Hannon GJ. MicroRNAs: small RNAs with a big role in gene regulation. Nat Rev Genet. 2004; 5:522-531.

20. Huntzinger E, Izaurralde E. Gene silencing by microRNAs: contributions of translational repression and mRNA decay. Nat Rev Genet. 2011; 12:99-110.

21. Di Leva G, Garofalo M, Croce CM. MicroRNAs in cancer. Annu Rev Pathol. 2014; 9:287-314.

22. Cioffi JA, Yue WY, Mendolia-Loffredo S, Hansen KR, Wackym PA, Hansen MR. MicroRNA-21 overexpression contributes to vestibular schwannoma cell proliferation and survival. Otol Neurotol. 2010; 31:1455-1462.

23. Erkan EP, Breakefield XO, Saydam O. miRNA signature of schwannomas: possible role(s) of "tumor suppressor" miRNAs in benign tumors. Oncotarget. 2011; 2:265-270. https://doi.org/10.18632/oncotarget.251.

24. Saydam O, Senol O, Wurdinger T, Mizrak A, Ozdener GB, Stemmer-Rachamimov AO, Yi M, Stephens RM, Krichevsky AM, Saydam N, Brenner GJ, Breakefield XO. miRNA-7 attenuation in Schwannoma tumors stimulates growth by upregulating three oncogenic signaling pathways. Cancer Res. 2011; 71:852-861.

25. Torres-Martin M, Lassaletta L, de Campos JM, Isla A, Gavilan J, Pinto GR, Burbano RR, Latif F, Melendez B, Castresana JS, Rey JA. Global profiling in vestibular schwannomas shows critical deregulation of microRNAs and upregulation in those included in chromosomal region 14q32. PLoS One. 2013; 8:e65868.

26. Eulalio A, Mano M, Dal Ferro M, Zentilin L, Sinagra G, Zacchigna S, Giacca M. Functional screening identifies miRNAs inducing cardiac regeneration. Nature. 2012; 492:376-381.

27. Sun Y, Cheng H, Wang G, Yu G, Zhang D, Wang Y, Fan W, Yang W. Deregulation of miR-183 promotes melanoma development via lncRNA MALAT1 regulation and ITGB1 signal activation. Oncotarget. 2017; 8:3509-3518. https:// doi.org/10.18632/oncotarget.13862.

28. Chen AC, Lee YL, Hou DY, Fong SW, Peng Q, Pang RT, Chiu PC, Ho PC, Lee KF, Yeung WS. Study of transforming growth factor alpha for the maintenance of human embryonic stem cells. Cell Tissue Res. 2012; 350:289-303.

29. Singh B, Coffey RJ. From wavy hair to naked proteins: the role of transforming growth factor alpha in health and disease. Semin Cell Dev Biol. 2014; 28:12-21.

30. Sasaki T, Nakamura T, Rebhun RB, Cheng H, Hale KS, Tsan RZ, Fidler IJ, Langley RR. Modification of the primary tumor microenvironment by transforming growth factor alpha-epidermal growth factor receptor signaling promotes metastasis in an orthotopic colon cancer model. Am J Pathol. 2008; 173:205-216.

31. Sasaki T, Hiroki K, Yamashita Y. The role of epidermal growth factor receptor in cancer metastasis and microenvironment. Biomed Res Int. 2013; 2013:546318.

32. Zehavi L, Avraham R, Barzilai A, Bar-Ilan D, Navon R, Sidi Y, Avni D, Leibowitz-Amit R. Silencing of a large microRNA cluster on human chromosome $14 \mathrm{q} 32$ in melanoma: biological effects of mir-376a and mir-376c on insulin growth factor 1 receptor. Mol Cancer. 2012; 11:44.

33. Zhu C, Li J, Ding Q, Cheng G, Zhou H, Tao L, Cai H, Li P, Cao Q, Ju X, Meng X, Qin C, Hua L, et al. miR-152 controls migration and invasive potential by targeting TGFalpha in prostate cancer cell lines. Prostate. 2013; 73:1082-1089.

34. Blair KJ, Kiang A, Wang-Rodriguez J, Yu MA, Doherty JK, Ongkeko WM. EGF and bFGF promote invasion that is modulated by PI3/Akt kinase and Erk in vestibular schwannoma. Otol Neurotol. 2011; 32:308-314.

35. Wang H, An H, Wang B, Liao Q, Li W, Jin X, Cui S, Zhang Y, Ding Y, Zhao L. miR-133a represses tumour growth and metastasis in colorectal cancer by targeting LIM and SH3 protein 1 and inhibiting the MAPK pathway. Eur J Cancer. 2013; 49:3924-3935.

36. Jin HY, Gonzalez-Martin A, Miletic AV, Lai M, Knight S, Sabouri-Ghomi M, Head SR, Macauley MS, Rickert RC, Xiao C. Transfection of microRNA mimics should be used with caution. Front Genet. 2015; 6:340.

37. Wang B, Ye N, Cao SJ, Wen XT, Huang Y, Yan QG. Identification of novel and differentially expressed MicroRNAs in goat enzootic nasal adenocarcinoma. BMC Genomics. 2016; 17:896. 
38. Anders S, Huber W. Differential expression analysis for sequence count data. Genome Biol. 2010; 11:R106.

39. Ioshikhes I, Roy S, Sen CK. Algorithms for mapping of mRNA targets for microRNA. DNA Cell Biol. 2007; 26:265-272.

40. Chen H, Zhang X, Zhang Z, Yang T, Wang Z, Wu H. The role of NF2 gene mutations and pathogenesis-related proteins in sporadic vestibular schwannomas in young individuals. Mol Cell Biochem. 2014; 392:145-152.
41. Patel AK, Alexander TH, Andalibi A, Ryan AF, Doherty JK. Vestibular schwannoma quantitative polymerase chain reaction expression of estrogen and progesterone receptors. Laryngoscope. 2008; 118:1458-1463.

42. Darzynkiewicz Z, Juan G. DNA content measurement for DNA ploidy and cell cycle analysis. Curr Protoc Cytom. 2001; Chapter 7:Unit 7.5. 